\section{VIVIENDA SOCIAL EN ECUADOR: VIOLENCIAS Y CONTESTACIONES EN LA PRODUCCIÓN PROGRESISTA DE PERIFERIAS URBANAS}

Gustavo Durán ${ }^{1}$, Manuel Bayón², Alejandra Bonilla Mena ${ }^{3}$, Michael Janoschka ${ }^{4}$

\section{Resumen}

La producción de vivienda de interés social fue por diez años uno de los emblemas de la política ecuatoriana de la Revolución Ciudadana presidida por Rafael Correa. Desde el caso de Ecuador, se analiza cómo los gobiernos progresistas aplicaron políticas en el ámbito de la vivienda social y el desarrollo urbano. Este artículo rastrea qué escalas de aplicación tuvieron estas políticas sociales respecto al neoliberalismo, desde el punto de vista de las violencias y contestaciones que el proceso suscitó. En Quito, las familias se organizaron para obtener las viviendas prometidas tras años

\section{SOCIAL HOUSING IN ECUADOR: TERRITORIAL VIOLENCE, CONTESTATIONS, AND PERIPHERAL URBAN SPACE PRODUCTION}

\author{
Gustavo Durán, Manuel Bayón, Alejandra
}

Bonilla Mena, Michael Janoschka

\section{Abstract}

Social housing production was one of the emblems of the Ecuadorian policy of the "Citizen Revolution" chaired by Rafael Correa for 10 years. From the case of Ecuador, it is analyzed how progressive governments applied policies in the field of social housing and urban development. This article tracks the scales of application these social policies had regarding neoliberalism, from the point of view of the violence and responses that the process provoked. In Quito, families organized to obtain the promised homes after years of waiting. In Guayaquil, police violence displaced entire neighborhoods to achieve 
de espera. En Guayaquil, la violencia policial desplazó barrios enteros para concretar la renovación urbana mediante un plan ecológico. En Portoviejo, a partir del terremoto de 2016, se reconvirtió el centro y se desplazó la vivienda a la periferia. Desde una metodología mixta histórica, espacial y cualitativa, este análisis muestra las limitaciones de las propuestas de los gobiernos progresistas y la organización popular para evitar desplazamientos hacia las periferias.

PALABRAS CLAVE: PERIFERIAS; VIVIENDA DE INTERÉS SOCIAL; VIOLENCIAS; CONTESTACIONES; ECUADOR.

Recibido: 2019-12-10

Aceptado: 2020-06-09 urban renewal through an ecological plan. In Portoviejo, from the earthquake of 2016, the center was converted, and the houses were moved to the periphery. From an historic, spatial, and qualitative methodology, this analysis shows the limitations of the proposals of the progressive governments, and the popular organization to avoid displacement towards the peripheries
KEYWORDS: PERIPHERIES; SOCIAL HOUSING; VIOLENCE; CONTESTATIONS; ECUADOR.

Received: 2019-12-10

Accepted: 2020-06-09
1 Facultad Latinoamericana de Ciencias Sociales, Ecuador, http://orcid.org/0000-0002-1930-0228. Correo electrónico: gduran@flacso.edu.ec

2 Facultad Latinoamericana de Ciencias Sociales, Ecuador, http://orcid.org/0000-0002-9450-9718. Correo electrónico mjimenezfl@flacso.edu.ec
3 Facultad Latinoamericana de Ciencias Sociales, Ecuador, http://orcid.org/0000-0001-7054-0481. Correo electrónico: apbonillam@gmail.com

4 Universität Leipzig, Alemania, http://orcid.org/0000-00026092-9052. Correo electrónico: michael.janoschka@unileipzig.de 


\section{La producción estatal de las periferias en el tránsito del neoliberalismo a los gobiernos progresistas}

Las políticas neoliberales implementadas en América Latina reforzaron la construcción de las ciudades como espacios más desiguales, segregados, fragmentados y excluidos. La configuración de las periferias ha sido central en el modelo de ciudad liberal, donde se destaca la producción de viviendas de interés social (en adelante VIS) en áreas formalizadas y en enormes áreas de exclusión informales. Esta dualidad hizo que la Producción Social del Hábitat (PSH) se convirtiera en la única posibilidad, para amplias franjas de población de sectores populares, de acceso a una vivienda (Hidalgo y Janoschka, 2014; Mattos, 2010). Esto implica una fuerte violencia estructural en la jerarquización de los espacios urbanos y en los derechos que fragmentan las distintas clases sociales urbanas. Así, este accionar público incita a procesos de desplazamiento como la forma más visible de territorialización de la exclusión urbana y, a su vez, destruye los hábitats construidos por las clases populares urbanas (Durán, Martí y Mérida, 2016).

En Ecuador, la crisis económica del 2000 implicó una explosiva migración a las ciudades que, ante un Estado colapsado, engrosó las áreas urbano-populares informales (Acosta, 2006). Los escasos planes de vivienda de la fase neoliberal se dieron bajo esquemas públicoprivados, donde se atendía a las clases sociales con cierta capacidad de gasto y endeudamiento, dejando fuera a los sectores más empobrecidos del país. En Quito y Guayaquil, las respuestas están muy vinculadas a los procesos de tomas de tierra periurbana, realizadas para poder generar asentamientos que hicieran efectivo el derecho a la vivienda (Sánchez, 2017).

La llegada de los gobiernos progresistas a la región implicó distintos niveles de reconfiguración de los Estados que, salvo casos específicos, generaron una modificación moderada del desarrollo territorial urbano resultante de la fase neoliberal. El caso de Ecuador se inscribe en esta ala más moderada de los gobiernos progresistas, siendo liderado por Rafael Correa y quedando plasmado de forma indirecta en su primer programa de gobierno (Alianza País, 2006). Las vías más moderadas están representadas por el neoestructuralismo promovido por la CEPAL, que postula como propuesta principal una mejor inserción en los mercados internacionales o el retorno del Estado como fuente de intervención (Stoessel, 2014). Sin embargo, esta visión neoestructuralista tuvo poca repercusión en la visión sobre el desarrollo urbano. Los balances, hechos por gobiernos de izquierdas en las ciudades, muestran más continuidades que rupturas respecto a los modelos territoriales 
neoliberales previos. Por lo que, los cambios se han centrado en el ámbito de la gestión pública de recentralización y no, en modelos públicoprivados, participativos, ni de construcción ciudadana (Carrión, 2015).

En Ecuador, esta visión se plasmó en el Plan de Desarrollo Territorial de la Secretaría Nacional de Planificación y Desarrollo, que se concretó con la reafirmación de la centralidad del Estado en la planificación territorial nacional y con una nueva forma de gestión donde primaba el poder de la administración central respecto a las administraciones locales (Burbano de Lara, 2015; SENPLADES, 2009). Esta nueva forma de gestión del territorio y sus escalas de intervención han reconfigurado las formas de violencia estatal y de organización de los barrios y comunidades para dar contestación a los proyectos desde sus lógicas.

En este artículo se analizan tres casos: los grandes proyectos de VIS y renovación urbana de las dos grandes ciudades del país (Guayaquil y Quito), junto con los pertenecientes a Portoviejo, uno de los epicentros de la afectación del terremoto de 2016 que asoló la costa ecuatoriana y donde la intervención en reconstrucción y VIS se convirtió en una política prioritaria del Estado. La comparación de las transformaciones territoriales y la subsecuente relación entre violencias y contestaciones dadas en estos tres casos de políticas de VIS permiten generar una discusión, insertada en la región latinoamericana, sobre los resultados de las nuevas formas de gobierno durante los gobiernos progresistas, sus alcances, límites y retos.

\section{Violencias y contestaciones urbanas en la producción de periferias}

En esta sección se realiza una aproximación a las violencias y contestaciones urbanas. Es necesario comprender que el espectro de lo que se considera como violencia se ha ampliado, y tiene múltiples dimensiones con distintos niveles de impactos que dificultan una especificidad del concepto. Ruiz (2012) ya analiza la violencia como característica de la experiencia urbana contemporánea y destaca dos tipos de violencias relevantes para nuestro estudio: institucional y estructural. La primera radica en el ejercicio de actos violentos, generado por instituciones públicas para mantener el poder de ciertos grupos, y tiene impacto en los servicios civiles urbanos: así, el caso de la vivienda. Por otro lado, la segunda violencia está vinculada al modelo hegemónico que replica desigualdad, y es ejercido sistemáticamente sobre la población. Esta segunda violencia se ha visto reforzada por las políticas neoliberales y Koonings y Kruijt (2007) la profundizan con la comparación de las violencias con espacios fragmentados, excluidos y marginados. 
Para comprender cómo se ha implantado la violencia, Montealegre y Rozas-Krause (2018), en su libro sobre disputar la ciudad, resaltan dos elementos: en primer lugar, la violencia inicia con el sometimiento, que en este caso es el sometimiento a una política de vivienda y de desarrollo urbano segregador; en segundo lugar, menciona que "si el sometimiento es una acción relacionada al poder jerárquico, la resistencia es inherente al poder ciudadano" (Montealegre y Rozas-Krause, 2018, p. 11). Consecuentemente, cuando la población atraviesa violencias, sean institucionales o estructurales, genera una contestación urbana que puede manifestarse de múltiples formas. Adicionalmente, las autoras rescatan que, una vez que se inicia el proceso de disputa, también se genera una memoria histórica para futuras resistencias.

Una de las dimensiones de la violencia es el desplazamiento de las clases populares de los centros de las ciudades para el recambio poblacional de clases más altas. Este ha atraído una gran cantidad de atención por parte de activistas, movimientos sociales y la academia, que se han dedicado a observar las violencias y las resistencias en el ámbito urbano (Janoschka, 2016; Smith, 1996). Bajo el fenómeno de gentrificación o de acumulación por desposesión se ha generado un amplio debate que ha logrado promover un enlace conceptual-político (Harvey, 2013).
En América Latina, la violencia de los desplazamientos ha sido especialmente estudiada en los centros de las megaurbes, donde ocurren procesos de gentrificación más cercanos al arquetipo que se da en otras grandes ciudades, aunque con procesos que también están relacionados con la informalidad del empleo y la posesión de la vivienda (Janoschka y Sequera, 2014). Los procesos de recambio poblacional, desplazamiento y violencias urbanas han sido poco tratados desde esta óptica en ciudades intermedias o en las periferias (Durán et al., 2016).

Una de las mayores violencias en las ciudades latinoamericanas se produce por la migración desde el campo, acelerada por la aplicación de medidas de ajuste estructural del neoliberalismo (Schiavo, Vera y Nogueira, 2014). Podríamos hablar entonces de que antes de los desplazamientos de población urbana hay una violencia que crea las ciudades por desplazamiento, que mediante un modelo de ciudad dual va a pasar a formar parte de las periferias informales. Este abandono total de grandes espacios de la ciudad por parte del Estado ha sido estudiado, a escala global, en las ciudades del sur (Davis, 2014). En ese ámbito, hay toda una serie de violencias superpuestas que deben ser miradas dentro de los procesos de violencia urbana asociados a los desplazamientos; por ejemplo, las violencias 
económicas originadas por la incertidumbre de la continuidad de la vivienda (Ortiz, 2011; Rodríguez, Saborido y Segovia, 2012), que llevan a una calidad del hábitat muy deficiente en relación con las áreas formales de la ciudad (Cruz-Muñoz e Isunza, 2017).

La construcción de vivienda estatal bajo el modelo neoliberal ha llevado, en distintos lugares de América Latina, a un desplazamiento de sectores populares hacia sucesivas periferias urbanas (Rodríguez y Sugranyes, 2005). El papel estatal se da también mediante sus políticas públicas de vivienda, cuando se decide a intervenir los sectores populares. La propia planificación de los barrios populares puede estar asociada a la enorme violencia de las reubicaciones obligadas en los nuevos espacios de "derecho a la vivienda" (Sánchez, 2017). Los estándares urbanos de estos programas, su localización, el tiempo hasta la entrega y la forma de entrega de las viviendas ha sido un fuerte campo de estudio en la región (Auyero y Berti, 2014). La conflictiva relación entre las violencias urbanas y los distintos niveles de la administración del Estado adquiere una importancia específica en América Latina. Las múltiples violencias, estructurales, sistémicas y cotidianas a las que son expuestas los barrios periféricos pueden provenir de la acción del mercado, del Estado, de los mismos sectores marginados e incluso de la naturaleza, a través de diversos orígenes, actores e instituciones involucradas (Marcuse, 1985; Slater, 2006; Smith, 1996).
Las resistencias emanan desde el derecho a la vivienda, al hábitat, al territorio, a la ciudad y a la no ciudad, en ocasiones desafiando a la expansión del capital de forma abierta y directa. Pero en otras ocasiones, la hegemonía del capital y los procesos mediante los cuales el Estado la materializa conducen a que los mecanismos de contestación se vean muy limitados en la esfera de la política formal (Bayón, 2016). Los sociólogos latinoamericanos han mostrado la importancia de comprender las complejidades de las estructuras de clase de las sociedades de todo el continente para emprender un análisis social y espacial. En particular, es crucial entender cómo el sujeto colectivo no solo se configura en términos económicos, sociales y culturales, sino también en términos étnicos, raciales y de género (García, 1982; Merklen, 2005). La forma espacial específica del hábitat popular, es decir, la manera en que las personas construyen y utilizan su entorno viviente, significa que la estructuración de clase da forma al contorno del espacio y lugar, que también se ha abordado en su dimensión insurgente (Caldeira, 2017; Duhau, 1998; Pelli, 2017).

Esto puede haber producido visiones a veces exageradas y románticas sobre las luchas emancipadoras y las supuestas fuertes tradiciones de autoorganización (Castoriadis, 1991; Zibechi, 2008). En este sentido, se ha demostrado que las disputas por el territorio generan dinámicas de resistencia a través de la producción de prácticas sociales cotidianas 
que ponen en duda el orden jerárquico de nuestras sociedades. Por ejemplo, las prácticas que interrumpen el proceso de acumulación de capital, como la ocupación ilegal, pueden constituir zonas temporalmente autónomas o territorios disidentes, que permiten a las comunidades controlar adecuadamente el espacio y el territorio, y desafiar los intereses del capital (Lopes de Souza, 2012). Si bien los procesos emancipadores pueden considerarse activos y políticamente conscientes, la experiencia de las luchas en América Latina también nos hace comprender que las contestaciones también pueden ser una práctica diaria inconsciente internalizada en los hábitos de las personas (Didi-Huberman y Miller, 2017).

Existe una gran potencialidad en observar los procesos de violencia y contestaciones urbanas desde un marco analítico que amplíe la perspectiva en dos sentidos. Por un lado, comprendiendo las formas de recambio poblacional más allá de los centros de las ciudades (mirando cómo se dan las violencias en las periferias informales de clases populares que tienen una conflictiva relación con el Estado y el mercado); por otro, considerando estas violencias como parte conjunta de sus contestaciones, desde un marco amplio que observe las movilizaciones y otros elementos más visibles, pero también aquellos menos evidentes que ocurren en la esfera de la vida cotidiana y de las relaciones barriales de los sectores populares, como válidas formas de disputa frente a la lógica de ordenamiento territorial del Estado.

\section{Metodología mixta para comparar las violencias y contestaciones territoriales}

La metodología busca realizar un análisis multiescalar de las políticas territoriales implementadas desde el marco de violencias y contestaciones para desentrañar cuáles han sido los elementos definitorios de las grandes políticas de vivienda en el período 2007-2017 en Ecuador. Para ello, se combinan métodos de análisis histórico-espacial, con métodos cualitativo-espaciales de análisis de políticas públicas. A su vez, se emplean instrumentos de recolección de datos, como: entrevistas con los habitantes de los barrios de estudio para analizar violencias promovidas por el Estado y el mercado y las correlativas contestaciones desplegadas por los barrios involucrados; entrevistas con los precursores públicos de los proyectos; y revisión documental del escenario.

Comprender la estructura del territorio, la forma en la que sus actores se han espacializado a lo largo de la historia y los poderes territoriales que lo disputan es fundamental para entender la producción de las formas urbanas actuales (Haesbaert, 2004). 
Ello comprende la documentación histórica, la representación estadístico-territorial de elementos cuantitativos y cualitativos de las estructuras económico-sociales y de las acciones y estrategias de diferentes sujetos. Se utilizan Sistemas de Información Geográfica (SIG) para territorializar los datos cualitativos recogidos mediante información primaria y secundaria que trabajan el nivel macro-escalar de las ciudades analizadas para mostrar los desplazamientos de sectores populares desde o hacialos barrios de estudio. Anivel meso-escalar, se analizan los planes de desarrollo territorial en la escala de los proyectos estudiados, la historia y lógicas de implementación de los proyectos en relación con la formulación de políticas públicas, complementado con entrevistas a profundidad de los funcionarios que tuvieron a su cargo los proyectos o declaraciones públicas sobre los mismos.

Desde un punto de vista cualitativo y microescalar, las entrevistas se centran en la trayectoria de vida y en las trayectorias organizativas de las dirigencias barriales, a fin de entender como la política de vivienda ha influenciado o condicionado la elección locacional de estas familias y sus consecuentes impactos. Las trayectorias de vida implican la reconstrucción del espacio-tiempo a partir de los recuerdos y la verbalización, de forma premeditada y proyectiva al mismo tiempo (Taylor y Bogdan, 1987). Di Virgilio entiende por trayectoria residencial "aquellas prácticas espaciales que involucran cambios en el lugar de residencia en la ciudad", considerando principalmente los aspectos vinculados a la localización en el territorio urbano (Di Virgilio, 2009, p. 235). Seleccionamos entre 15 y 20 entrevistas en cada espacio de estudio, así como recorridos y la observación participante como elemento para contextualizar y entender las lógicas y procesos de la vida en estos barrios; especialmente, para entender cómo las personas forman lugares (de significado) entre los espacios contestados y de violencia estructural.

\section{Resultados}

Para una primera aproximación, se destacan tres hitos de comparación de sus antecedentes. El primero es la crisis financiera de 2000-2007 cuando, de la mano de un feriado bancario y de la devaluación de la moneda oficial -sucrey su posterior dolarización, el país sufrió un brusco cambio y se vio inserto en los más altos índices de pobreza con migración externa, cuya expresión interna, en asentamientos irregulares, se concentró en Quito y Guayaquil, llegando en una escala mucho menor en Portoviejo. El segundo hito, son las diferencias en el gobierno local de las ciudades. Mientras en Guayaquil y Portoviejo los gobiernos locales fueron de partidos de derecha con un abierto enfoque neoliberal (2004 a la actualidad), en Quito dominaron, en el período de estudio, 
los gobiernos progresistas que ejecutaron VIS (2000-2014), con un recambio hacia un alcalde neoliberal en el período siguiente. El tercer hito es la presidencia de Rafael Correa por 10 años -de 2007 a 2017-, período en el que se centra el análisis del artículo. Un gobierno que se autodenominó progresista, que reconstruyó la institucionalidad del país y abogó por un cambio radical en la política territorial y de vivienda.

En cuanto a los casos de estudio, en Quito los programas de vivienda municipal en las periferias urbanas surgen de los gobiernos locales autodenominados de izquierda. En Guayaquil, un proyecto del Estado central progresista de regeneración y restauración ambiental condujo a desalojos hacia viviendas "llave en mano" buscando revertir la lógica neoliberal de la ciudad. Finalmente, en Portoviejo proliferan los proyectos VIS como resultado de la reubicación, guiada por el Estado central, posterior el terremoto de abril de 2016 (Figura 1).

\section{LAS POLÍTICAS PÚBLICAS DE PRODUCCIÓN DE NUEVAS PERIFERIAS URBANAS A PARTIR DE VIS}

El proyecto de Ciudad Bicentenario (CB), promovido por el Municipio de Quito, se dividió en dos etapas constructivas. Si bien la promoción de CB comenzó en 2005, no fue sino hasta 2009 cuando se construyeron las primeras viviendas. El proceso para acceder a la vivienda tuvo la alianza entre gobierno local y central, y se produce como se indica en la Figura 2.

Las familias de la primera etapa constructiva de Ciudad Bicentenario empezaron el proceso entre los años 2006 y 2008 y apenas tres años después accedieron a su vivienda. Las primeras 300 viviendas fueron entregadas en el año 2010. Si bien las promesas de entrega de vivienda se cumplen para ciertas familias, para otras se convierte en una contrariedad, dado que las familias acreedoras mantenían un arriendo de vivienda en otra parte de la ciudad. Esto generó que para muchas fuera inviable y desistieran del proyecto; mientras que para otras significó ajustar su economía y acudir a un préstamo.

"Quería tener mi casa para no estar paga y paga arriendo. Antes vivía en el Comité del Pueblo. Allá arrendaba, pagaba 200 USD y me subieron 250 USD el arriendo; y, pagaba acá, tocó hacer préstamo". (Ángel Yela, vecino de CB).

El modelo de violencia estructural del periodo neoliberal en la VIS se reprodujo con los materiales de construcción de baja calidad, la inhabitabilidad por falta de servicios básicos, por la falta de acceso con transporte y por la ausencia de legalización de escrituras. Si bien el municipio entregaba las viviendas en obra gris, con un área promedio de $38 \mathrm{~m}^{2} \mathrm{y}$ con posibilidad de construcción progresiva de $132 \mathrm{~m}^{2}$, el costo de construcción en altura era, para muchos, no factible. 
FIGURA 1. MOSAICO DE LOS MAPAS DE UBICACIÓN TRES CASOS. (A) QUITO (B) GUAYAQUIL (C) PORTOVIEJO.

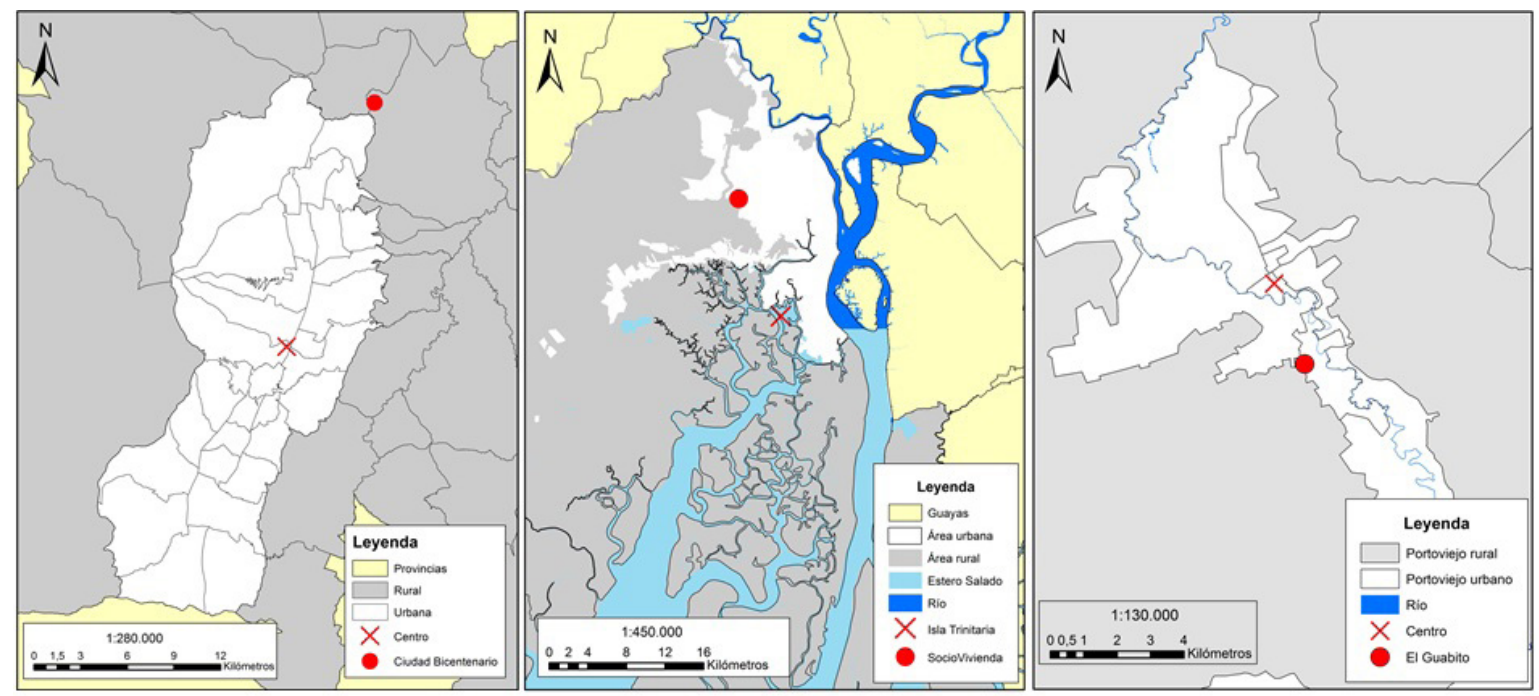

Fuente: Elaboración propia en base a coberturas del SNI

FIGURA 2. PROCESO PARA ACCEDER A LA VIVIENDA (FINANCIERAMENTE).

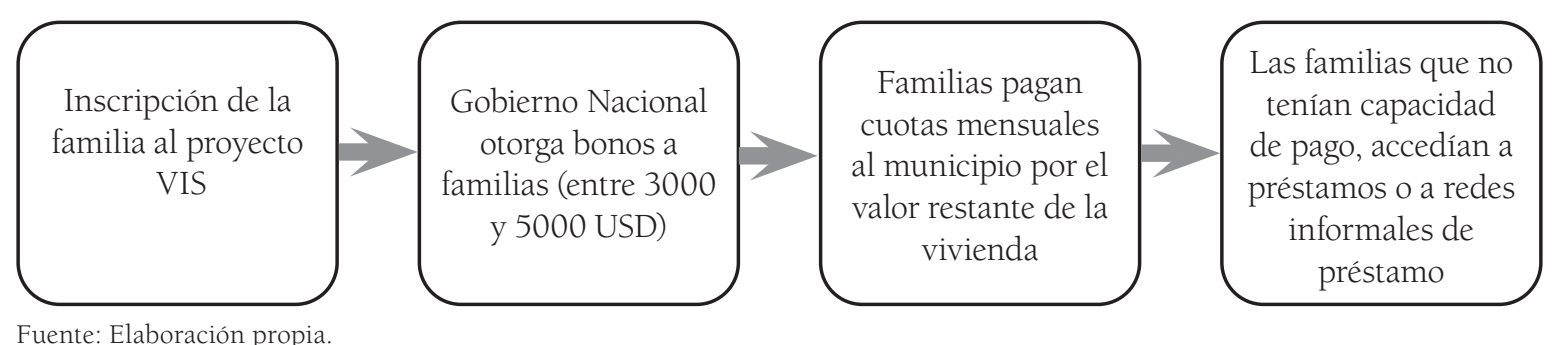


El cambio de gobierno local de Quito en 2014 cambia el panorama y refuerza el retorno al modelo neoliberal de VIS; y con ello, el problema se profundiza para la segunda etapa de CB. El cambio en el mecanismo de entrada a las viviendas y la falta de construcción de la etapa 2 hizo de la espera de las viviendas la mayor violencia contra las familias. Hasta el presente hay familias esperando sus viviendas.

"[...] ya habíamos pagado hace tres años atrás, antes de que nos hagan la entrega, entonces cómo así les voy a pagar por algo que yo ya pagué, yo ya cumplí con lo mío, hice un convenio por 9700 USD y terminé pagando 13500 USD. Entonces yo ya les pagué más, ya no es algo que a mí me compete, fue el incumplimiento de ellos". (Aracely, vecina de la etapa 2 de CB).

En Guayaquil, la producción de VIS en la periferia urbana se da por intervención estatal en el 2011, bajo el nombre de SocioVivienda. Guayaquil es una de las ciudades más desiguales del Ecuador, que ha sido gobernada por un partido de derecha por más de 20 años (Sánchez, 2017). Cuando Correa sube al poder, declara su poca empatía con el modelo conservador de Guayaquil y opta por ejercer presión:

"Vamos a intervenir en Guayaquil con el 'Plan Guayaquil Ecológico', una ciudad con una gran problemática ambiental por la nefasta política social cristiana que ha permitido que esteros y montañas sean invadidos y destrozados, para convertirla en la ciudad ecológica que siempre debió ser, un modelo exitoso de igualdad y justicia" (Gobierno Nacional, 2010).

Sin embargo, el ideal de ciudad que él planteaba no tomaba en cuenta el habitar de las personas del Estero Salado, que encontraban ese espacio como un hogar y que fue levantado mediante PSH (Sánchez, 2017). Lo que se produce entonces es una renovación urbana, escondida bajo la protección de la Reserva Ecológica de Manglar en las riberas del Estero Salado y con una política pública de vivienda que otorga al sector privado la producción masiva de viviendas, del mismo prototipo y con bajos estándares habitacionales (Figura 3).

Desde el gobierno nacional se construyó el proyecto VIS SocioVivienda II, cuyos usuarios fueron familias previamente desalojadas mediante violentos operativos policiales; familias que perdían sus viviendas y pertenencias solo 15 minutos después del aviso. De acuerdo con el reporte del proyecto de Ministerio de Vivienda y Desarrollo Urbano (MIDUVI) del 2015, desde el año 2011 hasta diciembre del 2014, se desplazaron 3.478 familias y se esperaba que para el 2015 llegara a 8.175 familias. El modelo para desalojar a las familias y reubicarlas en los proyectos VIS se presenta en la Figura 4.

Los problemas y conflictos que se producen son fuertes. Por un lado, el Ministerio del Ambiente (MAE) se enfoca en restaurar el Estero y el MIDUVI en construir viviendas para 
FIGURA 3. CRONOLOGÍA DEL PROYECTO GUAYAQUIL ECOLÓGICO EN EL ESTERO SALADO.

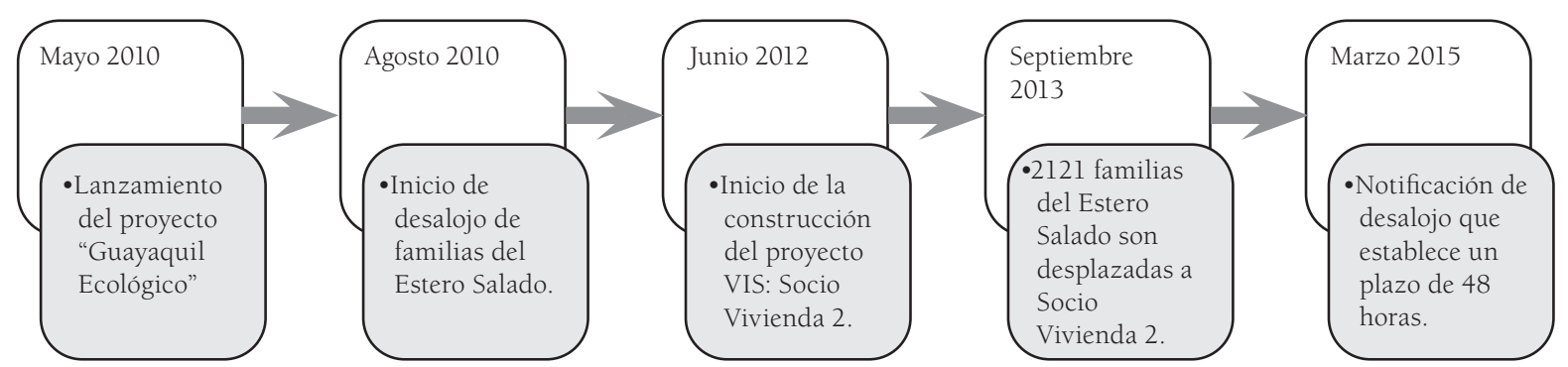

Fuente: Elaboración propia.

FIGURA 4. PROCESO IMPLEMENTADO POR EL MIDUVI PARA EL DESALOJO.

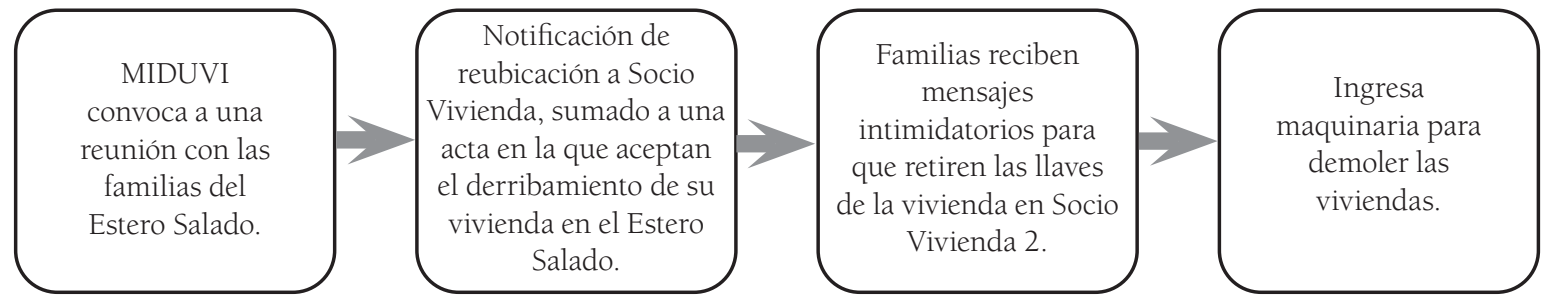

Fuente: Elaboración propia.

FIGURA 5. PROCESO IMPLEMENTADO POR EL COMITÉ DE RECONSTRUCCIÓN PARA OTORGAR LAS VIS.

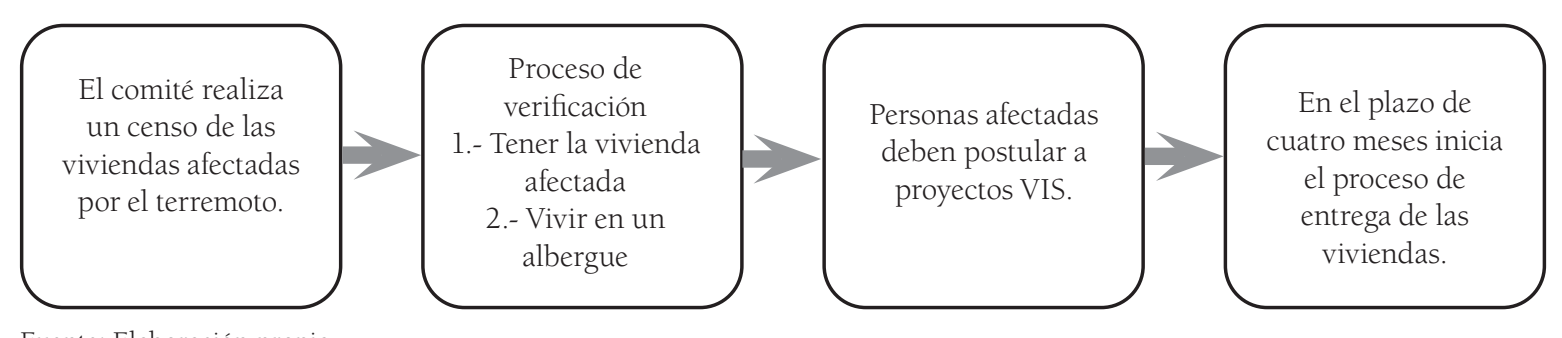

Fuente: Elaboración propia. 
los desplazados; pero, por otro lado, están los habitantes que han construido su habitar en el Estero y no quieren desplazarse, más aún si lo que se oferta son viviendas de producción en masa en zonas alejadas (Portaluppi, 2020).

Al momento de visitar los proyectos, se reconoce que Socio Vivienda es el emblema de una política de vivienda fallida. En primer lugar, las nuevas viviendas no cumplen los mínimos estándares de construcción ni de tipología (la superficie promedio de vivienda es de $5,2 \mathrm{~m}^{2} /$ persona, y las familias no cuentan con su propiedad). En segundo lugar, las familias reubicadas fueron despojadas de su vivienda, que eran espacios de encuentro, de redes familiares y comerciales, y de habitar. En tercer lugar, se refuerza el patrón de segregación espacial y se conduce a las familias a un escenario de exclusión y de guetto, donde sus habitantes temen las mínimas interacciones cotidianas y sus condiciones de vida se han precarizado, anhelando por encontrar una vivienda en otro sector.

En el caso de Portoviejo, la VIS se encamina a reubicar a personas desplazadas por el terremoto del 16 de abril de 2016. Mediante el decreto ejecutivo $N^{\circ} 1004$ del 26 de abril se creó el Comité de Reconstrucción y Reactivación Productiva y del Empleo en las zonas afectadas por el terremoto, asumiendo el Estado central un rol protagónico, con el MIDUVI construyendo viviendas para las personas damnificadas. Uno de los proyectos es El Guabito, con 600 viviendas en la periferia urbana. El proceso para obtener la vivienda se indica en la Figura 5.

La construcción se dio en tiempo récord, por lo que las violencias no están asociadas al tiempo, sino a la habitabilidad. Los bloques de vivienda, de aproximadamente $12 \times 13 \mathrm{~m}$, comprenden cuatro viviendas: dos en planta baja y dos en planta alta, ambas sin posibilidad de crecimiento progresivo. El tamaño reducido y el bajo estándar constructivo son algunos de los problemas.

"[...] Se están cayendo los troncos, las escaleras se están cayendo, es decir, esas no las hicieron como deberían [...] La verdad es que nosotros vinimos y las casas ya estaban hechas, las vinimos a conocer cuando las entregaron. La idea de nosotros no era que nos dieran acá". (Juan Carlos y su esposa, vecinos de EG).

Otro de los problemas que existe en este tipo de proyecto es la ausencia de derechos de propiedad. Para algunos moradores esta es la causa de la degradación y del nulo sentido de apropiación del espacio por parte de sus vecinos, sumado a la inseguridad, producto de la delincuencia y consumo de drogas. Adicionalmente, la localización no ayuda; el $80 \%$ de las personas reubicadas vivían en el centro de la ciudad, por lo que El Guabito les significa un problema de accesibilidad, un problema para entablar relaciones sociales y un problema de inserción en el mercado laboral. Sin embargo, el actual gobierno local observa la 
reconstrucción como la posibilidad de eliminar el comercio popular del centro. Los pobladores lo describen así:

"El cambio fue recontra brusco. Nosotros estábamos en el centro de la ciudad, caminábamos dos cuadras y teníamos todo [...] Usted ve muchas de las familias caminando desde aquí a Portoviejo, hay más de $2 \mathrm{~km}$, y lo hacen porque ni siquiera tienen para el colectivo [...]. En la reconstrucción no han hecho nada por nosotros [...] Aquí ya el alcalde tiene que darse cuenta de que, el centro es el corazón de Portoviejo [...] que aquí hay que hacer un centro comercial para que eso impulse nuevamente la activación de Portoviejo". (Juan Carlos y su esposa, vecinos de $E G)$.

\section{LAS FORMAS DE CONTESTACIÓN A LAS VIOLENCIAS DE LA INTERVENCIÓN ESTATAL}

Una vez descrito el proceso de implementación y la producción del espacio periférico urbano mediante VIS en los tres casos seleccionados, este apartado realiza un análisis comparado para mostrar las continuidades y rupturas a partir de tres ejes: desde la micro-escala del individuo, a partir de los desalojos; posteriormente a escala de vivienda; y, finalmente, a la escala ciudad, para territorializar las violencias ejercidas por el Estado durante el período progresista de Ecuador. En un marco general, tal y como lo menciona Ruiz (2012), este tipo de violencia responde a un modelo hegemónico que replica desigualdad y se ampara en políticas de vivienda para ejercer un sometimiento sobre la población. Por otro lado, este apartado también analiza cómo la reconfiguración de las políticas públicas ha implicado acciones de contestación basadas en la mitigación de las violencias en el ámbito de los desalojos y la construcción de VIS.

\section{Contestaciones ante LAS VIOLENCIAS DE VIVIENDAS PRECARIAS}

Las viviendas construidas de Socio Vivienda y El Guabito tienen estándares constructivos sin una dignidad mínima para sus habitantes. La imposibilidad de crecimiento vertical u horizontal agrava la situación; en muchas de las viviendas visitadas hay un uso de las salas como dormitorios. No se han entregado las escrituras de propiedad a las familias, generando poco apego y frenando la inversión en mejoras de los departamentos entregados. A pesar de que en Ciudad Bicentenario el espacio de la vivienda fue mejor planteado, ahí las violencias están asociadas a la no entrega de las viviendas y a las formas de endeudamiento que asumieron las familias, violencias que surgen desde la dimensión institucional (Taller con Ciudad Bicentenario, 2019).

Ante la violencia de la política de vivienda de producción en masa, el espacio íntimo se convierte en un espacio de contestación en la vida cotidiana. Mediante ampliaciones de cobertizos, aprovechando los retiros, inventando 
un sinnúmero de opciones constructivas, las familias hacen frente a la escasez de espacio en las viviendas. No solo en tamaño, también en las funciones de las habitaciones, en las que aparecen pequeñas tiendas que se convierten en núcleos de relación vecinal. Aunque hay supuestas regulaciones que limitan este tipo de usos y actividades, la voluntad de control efectiva del Estado es nula, por lo que se van generando espacios de relación y economías de subsistencia entre las viviendas.

\section{Contestaciones ante La VIOLENCIA del hábitat de LOS VIS}

Los tres casos comparten el hecho de que el sector público provee las viviendas quedando, sin embargo, un sinfín de necesidades fundamentales que no están contempladas ni construidas. La violencia de este apartado responde a la construcción de un espacio aislado y sin relaciones sociales que anticipa, para la institución, un entorno ideal para un fácil sometimiento. Por esta razón se ha dado otra escala de contestación en la construcción del hábitat barrial (Figura 6); es decir, la llegada de las familias a lugares que aún requieren una serie de infraestructuras mínimas ha sido objeto de mediación por parte de las dirigencias barriales. Las primeras familias que llegaron a Ciudad Bicentenario solo tenían la vivienda y en la memoria se guardan la nula accesibilidad, la ausencia de agua y alcantarillado, y el lodazal aislado en el que habitaban. Una década después, Ciudad Bicentenario solo cuenta con el $10 \%$ de equipamientos públicos planificados por el Municipio.

El Guabito y Socio Vivienda comparten un fenómeno de guettización propio del neoliberalismo, donde la dejadez del Estado, una vez terminadas las viviendas, ha sido prácticamente total. En ambos espacios, sus dirigencias manifestaron que la venta de droga en el barrio era uno de sus principales problemas, estrechamente asociada a la ausencia de posibilidades laborales producida por el desplazamiento. De esta forma, la ausencia de programas sociales o espacios comunitarios ofrece pocas alternativas para rehacer las relaciones al interior de los barrios. Ante todo lo expuesto, los reclamos para la construcción de vías, alcantarillado o veredas en Ciudad Bicentenario, junto a la pelea por las áreas verdes en El Guabito o la mejora de los servicios básicos y equipamiento en SocioVivienda, han sido parte de las gestiones que las dirigencias barriales afrontaron inicialmente. Cabe destacar que, hasta la actualidad, las dirigencias barriales son el tipo de organización que se ha mantenido en los barrios analizados. 
FIGURA 6. MOSAICO DE LOS TRES

PROYECTOS Y SUS EQUIPAMIENTOS

(A) CIUDAD BICENTENARIO (B)

SOCIOVIVIENDA (C) EL GUABITO.

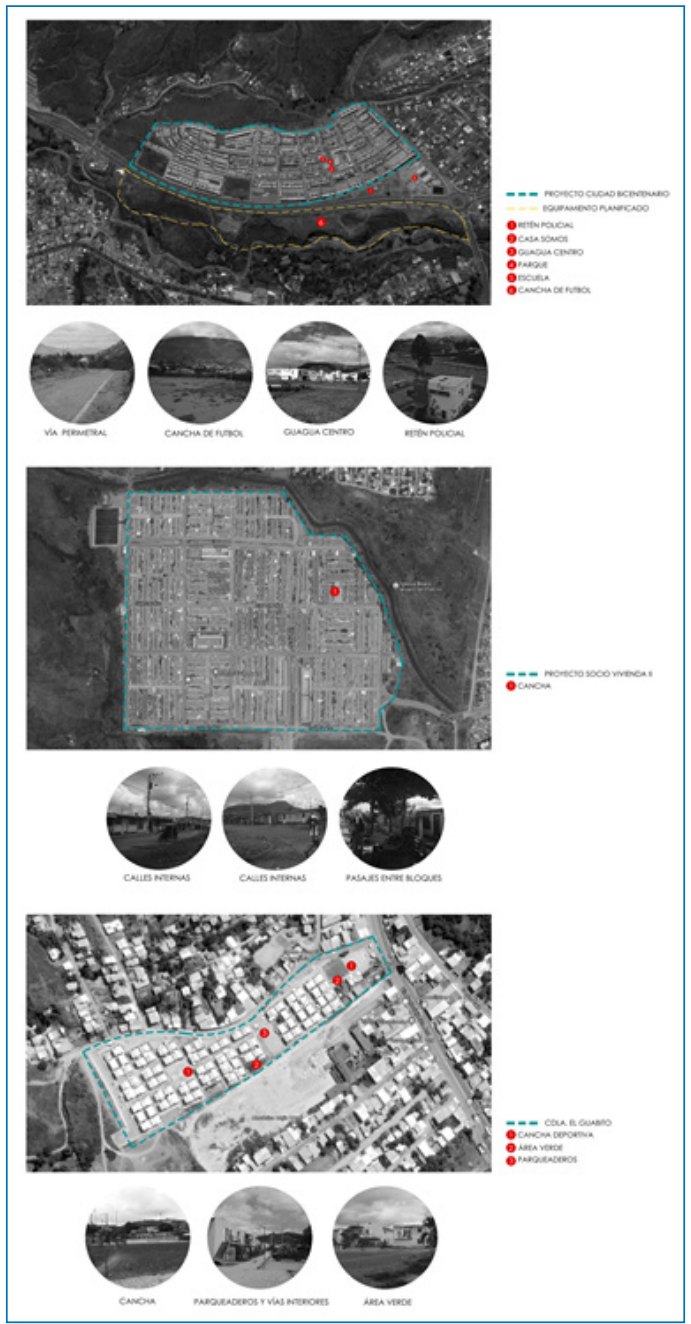

Fuente: Elaboración de Johanna Villavicencio. 


\section{Contestaciones ante las VIOLENCIAS DE LOS DESPLAZAMIENTOS Y REUBICACIONES}

En los tres lugares analizados, los desplazamientos internos se producen en las ciudades desde áreas relativamente centrales hacia las nuevas periferias producidas desde el Estado (Figura 1). Siguiendo el argumento de Janoschka, Smith y Harvey, al desplazar a los estratos populares a viviendas sociales ubicadas en las periferias se replica una dimensión de la violencia: la acumulación por desposesión. Este movimiento hacia áreas muy distantes destruye las dinámicas de vida, las relaciones y los sentidos de pertenencia en el espacio. Ante estas violencias, se producen diferentes contestaciones que varían en función de la forma de tejido social producida en los barrios.

En Guayaquil, las familias fueron desalojadas de sus lugares de vida -en áreas cercana a la ciudad consolidada- para ser trasladados hasta una zona rural desconectada. La violencia física más explícita ha sido desplegada por el Estado central en este caso, donde las familias fueron evacuadas por la Policía Nacional y el Ejército con escasos minutos de aviso previo, procediendo luego a derrocar las viviendas (Comité Permanente por la Defensa de los Derechos Humanos, 2016). Bajo este escenario, las familias desalojadas violentamente o bajo la amenaza acudieron al Comité de Derechos Humanos, que da seguimiento a los casos, articulando a las familias afectadas. Posteriormente, se dieron acciones de protesta para frenar las peores expresiones de violencia en los desalojos: en Isla Trinitaria y el Suburbio, hubo movilizaciones en formas de marchas en el centro de Guayaquil, que hicieron que numerosos tramos previstos de Guayaquil Ecológico no fueran desalojados. La cada vez mayor atención mediática, la visibilidad de la violencia aplicada por la policía y los pronunciamientos de la Relatora Especial de la ONU de Vivienda Adecuada, Raquel Rolnik, hicieron una presión creciente sobre el Estado ecuatoriano, en vísperas de la celebración de la Conferencia de ONU-Hábitat III en Quito.

En Quito, la promoción de VIS es en el norte de la ciudad, en áreas fuera del perímetro urbano (por la restricción de capacidad de pago en áreas con mayor accesibilidad). Existen dos escenarios diferenciados por las etapas constructivas: la Etapa I se organizó para reclamar por el acceso a servicios y equipamientos; las familias de la Etapa II, sin embargo, se organizaron para luchar contra la demora en la entrega de sus viviendas: a pesar de que las viviendas estaban construidas, el Municipio no autorizaba la entrada de las familias, que tenían dificultad para organizarse por no conocerse. La organización se creó a partir de auto-convocarse mediante afiches por la ciudad, buscando un espacio de encuentro para generar una asamblea. Un grupo promotor de esta reunión, formado mayoritariamente por mujeres, logró, mediante presión al Municipio, un listado de las personas y convocaron la primera asamblea: la asistencia 
fue protagonizada por 1000 familias afectadas que aprobaron la conformación de una asociación. Una vez organizados, la asociación de los afectados de la Etapa II boicoteó eventos del alcalde de Quito tras la paralización de las obras de Ciudad Bicentenario o cuando el Municipio anunció el aumento del precio de las viviendas. Un grupo de mujeres comenzó a acudir con pancartas a la inauguración de obras terminadas en el norte de Quito y convocaron una marcha hasta el Municipio que acabó en la firma de acuerdos con la Vicealcaldesa para la construcción de las viviendas.

En Portoviejo, el terremoto de 2016 acaba con los lugares de vida y de trabajo de la población popular del centro de la ciudad, dejando a las familias sin expectativas de retorno a las áreas centrales por la ausencia de una reconstrucción efectiva. La acción de destrucción y desalojo de las viviendas fue por el terremoto, seguido por una evacuación militar de la zona centro y por una limitación de regreso indefinida que impidió a las familias reasentarse en las áreas que habitaban. La actuación del Estado en medio de la gestión post-terremoto hizo que no se diera una organización de las personas afectadas como tal. Una vez llegaron a El Guabito, se eligió una directiva, lo que ocurrió también en los otros dos casos de análisis. Tomando en cuenta la violencia estructural de desigualdad y segregación espacial impuesta al desplazar a las familias a la periferia urbana, alejándolas del centro de la ciudad en el que antes habitaban, la capacidad de autoorganización ha sido muy limitada. En los espacios de vida del centro de la ciudad, en el que habitaban las familias desplazadas, emergieron como actores sociales las asociaciones de comerciantes, que buscan revivir la vocación comercial y popular del Centro Histórico. Pese a que han logrado el apoyo de arquitectos de la ciudad para la formulación de propuestas de planificación y diseño de centros comerciales, su capacidad de incidencia ha sido limitada en la búsqueda de reactivación de la actividad económica que tanto demandan los habitantes de sectores como El Guabito.

\section{AusenCia de CONTESTACIONES EN LA DISPUTA DE LA CENTRALIDAD}

Hay una violencia de deslocalización, dado que la política de vivienda estatal no ha buscado formas de disputa del centro de las ciudades para las VIS. Los lugares de partida de las familias de Quito y Guayaquil están sujetos a violencias estructurales de la composición segregada de las ciudades; a diferencia de Portoviejo, donde las clases populares habían logrado instalarse en el centro económico y administrativo. En la escala de la producción del espacio urbano, los tres casos muestran una evolución temporal en la que el Estado progresista solo ha mirado la escala del conjunto de la ciudad. Ciudad Bicentenario comienza bajo una gestión empresarialista, pese a estar enmarcado en un gobierno progresista, y termina en la misma 
lógica. Por su parte, el proyecto Guayaquil Ecológico supone una planificación urbana en una escala suprabarrial, tratando de modificar los patrones de crecimiento informal mediante VIS en el noroccidente de la ciudad. No deja de ser paradójica una intervención del MIDUVI y MAE en el ámbito urbano siendo el ordenamiento urbano una competencia municipal. En la reconstrucción post-terremoto se va un paso más allá y se generan entes de planificación territorial y de ejecución de la política multisectorial mediante la Secretaría de Reconstrucción. Los programas de vivienda de Portoviejo son, así, parte de una renovación urbana integral a escala ciudad donde la construcción de VIS en las periferias urbanas es parte de una visión de un nuevo centro gentrificado.

Como parte de esta escala, se observa que la diversa composición política del gobierno nacional progresista y de los gobiernos locales conservadores no ha implicado una forma diferenciada de ver el conjunto de las ciudades, dado que los paradigmas de renovación urbana y VIS no tuvieron modificaciones sustanciales respecto a la fase neoliberal. Tal vez en Quito se puede mencionar una modificación en la forma de gestión de las VIS, pero no una nueva concepción de estas. Por lo tanto, en estos proyectos se aplicó una política vertical desde el Estado, donde las familias, organizaciones y barrios afectados, fueron observados como meros usuarios de la política pública.

\section{EL TIEMPO COMO ESCALA EN DISPUTA}

Por último, esta comparación arroja el tiempo como una dimensión a ser considerada en esta mirada multi-escalar. El tiempo de la vida en los sectores populares ha sido violentado por los tiempos de la intervención del Estado. En Ciudad Bicentenario, con un tiempo de hasta 15 años de espera para que las familias ejerzan su derecho a la vivienda. En SocioVivienda los tiempos de los desalojos son rápidos, y no tienen en cuenta la territorialidad de los sectores populares, en una lógica que desprecia los tiempos de la consolidación de las viviendas. En Portoviejo, 50 segundos de vibración de la tierra son seguidos de tres años de exclusión en las periferias. Siguiendo el argumento de Auyero y Berti (2014), la doble violencia temporal a los sectores urbano-populares radica en tiempos de espera burocráticos para ser merecedores de una VIS y, por otro lado, el Estado no considera todo el tiempo socialmente necesario para construir su hábitat.

Como conclusión de este apartado, en el ámbito de desarrollo de VIS, no pudimos observar una mayor modificación de la concepción de la política pública de los gobiernos nacionales y locales progresistas, ni tampoco ver que se lograra salir de los círculos neoliberales de diseño de viviendas, como consecuencia de la dejadez en la producción del hábitat o de la ausencia de mecanismos de participación social. Sin embargo, no se puede afirmar que haya una completa continuidad en esta fase, 
ya que la recentralización del Estado supuso escalas de actuación que no acontecieron durante el período neoliberal previo, en el que comenzó a considerarse a la VIS como parte de una planificación a escala de ciudad. La articulación de estas tendencias explica que las violencias inherentes a la fase neoliberal de producción urbana se han reconfigurado en nuevas escalas e intensidades.

Tomando en cuenta el impacto de la violencia en diferentes escalas, es también necesario mencionar que, aunque los individuos y las familias se han organizado para contestar a las violencias percibidas a escala de la vivienda, el barrio y los desplazamientos, no se han visto contestaciones en la escala de ciudad. Estos tres casos muestran que las contestaciones lograron detener las violencias más explícitas. Sin embargo, no ha habido un cuestionamiento sobre la producción estatal de los barrios de VIS, ni sobre su ubicación en la ciudad, ni sobre su lógica constructiva, ni la formulación de propuestas que permitan salir de la visión empresarialista y neoliberal de su gestión e implementación territorial.

\section{Conclusiones}

El artículo muestra las formas de intensificación de las violencias urbanas que se han dado bajo un Estado planificador que logra re-centralizar y fortalecer sus capacidades para producir el espacio urbano. La fase de gobierno del ciclo progresista en Ecuador, que buscaba dejar atrás la formulación de planificación territorial del neoliberalismo, no logró formular ni aplicar formas de construcción de VIS que plantearan "modelos progresistas". El espacio de la ciudad para las VIS sigue siendo la periferia fuera del perímetro urbano, con viviendas que buscan el menor coste posible, implicando el uso de materiales deficientes y tiempos dilatados en la construcción y titularización.

Pese a que hay una continuidad en la concepción de construcción de VIS, la mayor capacidad del Estado central de poner en marcha políticas territoriales implicó una reformulación de las formas de violencias. Las periferias informales pasaron a ser intervenidas con procesos de desplazamiento masivo que provocaron unas formas de violencia física estatal sin precedentes en la historia reciente de Ecuador. El paradigma neoliberal de reforma urbana se anuda con la capacidad efectiva del estado planificador para aplicar violencia en proporciones mayores, necesarias para la reconfiguración de los espacios centrales y periféricos en tiempos acelerados.

Las contestaciones de los barrios afectados por estas violencias han tenido sus hitos más visibles contra la plasmación de las violencias más extremas de desalojo o de no entrega de viviendas. Pese a estar muy localizadas en el tiempo, logran que los aspectos más violentos 
de estos proyectos sean mitigados, por lo que la capacidad planificadora del Estado -bajo el gobierno progresista de Ecuador- no fue total. Hay que remarcar que las contestaciones de estos barrios no han puesto en duda la forma de gestión y plasmación de las políticas de VIS, y por ello, tras los hitos de movilización, se han terminado focalizando en la escala de la mediación barrial para lograr mejoras del hábitat, o la reconstitución del espacio íntimo de la vivienda.

El balance de este estudio comparado de tres casos muestra cómo el gobierno progresista de Ecuador dedicó una muy escasa atención a la generación de modificaciones sustantivas de los procesos de urbanización, generando políticas verticales para sectores populares urbanos, en unas intervenciones que desconocieron la lógica y las realidades de los barrios construidos de manera informal mediante procesos de PSH. Bajo una planificación centralizada del espacio urbano, el estado multiplicó las violencias y su escala de aplicación, sin disputar el sentido de la construcción de la ciudad neoliberal.

\section{Referencias bibliográficas}

Acosta, A. (2006). Breve historia económica del Ecuador. Quito: Corporación Editora Nacional.

Alianza País. (2006). Plan de gobierno del Movimiento PAIS 2007-2011. Quito: Alianza País.

Auyero, J. y Berti, M. (2014). La violencia en los márgenes. Una maestra y un sociólogo en el conurbano bonaerense. Buenos Aires: Katz.
Bayón, M. (2016). Comunidades rurubanas de Quito: entre el empresarialismo y el derecho a la ciudad. Íconos. Revista de Ciencias Sociales, 56, 103-22.

https://doi.org/10.17141/iconos.56.2016.2068

Burbano de Lara, F. (2015). La izquierda en el gobierno de Quito cuando la ciudad sucumbe frente al centro y la nación. En F. Carrión y P. Ponce (Eds.), El giro a la izquierda: los gobiernos locales de América Latina (pp. 291-332). Quito: Quinta Avenida.

Caldeira, T. (2017). Peripheral urbanization: Autoconstruction, transversal logics, and politics in cities of the global south. Environment and Planning D, Society and Space, 35(1), 3-20.

https://doi.org/10.1177/0263775816658479

Carrión, F. (2015). El giro a la izquierda: los gobiernos locales de América Latina. En F. Carrión y P. Ponce (Eds.), El giro a la izquierda: los gobiernos locales de América Latina (pp. 21-56). Quito: Quinta Avenida.

Castoriadis, C. (1991). Philosophy, politics, autonomy. Nueva York: Oxford University Press.

Comité Permanente por la Defensa de los Derechos Humanos. (2016). Informe sobre desalojos forzosos en Guayaquil. Período: 2013 - 2016. Guayaquil: Autor.

Cruz-Muñoz, F. e Isunza, G. (2017). Construcción del hábitat en la periferia de la Ciudad de México. Estudio de caso en Zumpango. EURE, 43(129), 187-207.

http://dx.doi.org/10.4067/S0250-71612017000200009

Davis, M. (2014). Planeta de ciudades miseria. Madrid: Akal. 
Di Virgilio, M. (2009). Trayectorias residenciales y estrategias habitacionales entre familias de sectores populares y medios residentes en el área metropolitana de Buenos Aires, Argentina". En J. Erazo (Ed.), Inter/secciones urbanas: origen y contexto en América Latina (pp. 233-257.) Quito: FLACSO.

Didi-Huberman, G. y Miller, C. (2017). Critical image/ Imaging critique. Oxford Art Journal, 40(2), 249-61.

\section{https://doi.org/10.1093/oxarti/kcx027}

Duhau, E. (1998). Hábitat popular y política urbana. México D. F.: Universidad Autónoma Metropolitana.

Durán, G., Martí, M., y Mérida, J. (2016). Crecimiento, segregación y mecanismos de desplazamiento en el periurbano de Quito. Íconos, (56), 123-146. https://doi.org/10.17141/iconos.56.2016.2150

García, N. (1982). Las culturas populares en el capitalismo. La Habana: Casa de las Américas.

Gobierno Nacional. (2010, 20 de febrero). Enlace ciudadano 160 - 20 [Programa de televisión].

Haesbaert, R. (2004). O mito da des-territorialização. Rio de Janeiro: Bertrand.

Harvey, D. (2013). Ciudades rebeldes. Madrid: Akal

Hidalgo, R. y Janoschka, M. (2014). La ciudad neoliberal. Gentrificación y exclusión en Santiago de Chile, Buenos Aires, Ciudad de México y Madrid. Santiago de Chile: Instituto de Geografía, Pontificia Universidad Católica de Chile.
Janoschka, M. (2016). Gentrificación, desplazamiento, desposesión: procesos urbanos claves en América Latina. Revista INVI, 31(88), 27-71.

http://dx.doi.org/10.4067/S0718-83582016000300002

Janoschka, M. y Sequera, J. (2014). Procesos de gentrificación y desplazamiento en América Latina, una perspectiva comparativista. En J. Michelini (Ed.), Desafios metropolitanos: un diálogo entre Europa y América Latina (pp. 82104). Madrid: Catarata.

Koonings, K. y Kruijt, D., (Eds.). (2007). Fractured cities: social exclusion, urban violence, and contested spaces in Latin America. New York, Estados Unidos, Zed Books.

Lopes de Souza, M. (2012). Challenging heteronomous power in a globalized world. En S. Krätke, K. Wildner y S. Lanz, (Eds) Transnationalism and urbanism. Nueva York: Routledge.

Marcuse, P. (1985). Gentrification, abandonment, and displacement: Connections, causes, and policy responses in New York City". Journal of Urban and Contemporary Law, 28(1), 195-240.

Mattos, C. d. (2010). Globalización y metamorfosis urbana en América Latina. Quito: Distrito Metropolitano.

Merklen, D. (2005). Pobres ciudadanos. Las clases populares en la era democrática (Argentina, 1983-2003). Buenos Aires: Gorla.

Montealegre, P. y Rozas-Krause, V. (Eds.). (2018). Disputar la ciudad: sometimiento, resistencia, memorialización y reparación. Talca, Bifurcaciones. 
Ortiz, E. (2011). Producción social de la vivienda y el hábitat. Bases conceptuales y correlación con los procesos habitacionales. México D. F.: HIC.

Pelli, V. (2017). El mejoramiento habitacional de los asentamientos espontáneos. Cuaderno Urbano, 2(2), 57-63.

http://dx.doi.org/10.30972/crn.222054

Portaluppi, C. (2020). Nuevos derechos sin nuevas políticas urbanas. Estudio de caso del componente restauración ecológica del Estero Salado del proyecto Guayaquil Ecológico 2010-2017. (Tesis de Maestría, sin publicar). FLACSO, Argentina.

Rodríguez, A., Saborido, M., y Segovia, 0. (2012). Violencias en la ciudad neoliberal: Santiago de Chile. Santiago de Chile: Ediciones SUR.

Rodríguez, A. y Sugranyes, A. (2005). El problema de vivienda de los con techo. En A. Rodríguez y A. Sugranyes, (Eds.). Los con techo: un desafío para la vivienda social. Santiago de Chile: Ediciones SUR.

Ruiz, J. (2012). Violencias en la periferia de Santiago. La población José María Caro. Revista INVI, (27) $74,249-285$.

http://dx.doi.org/10.4067/S0718-83582012000100008

Sánchez, P. (2017). La ciudad desde abajo vs. la ciudad desde arriba. Contradicciones entre la lógica de la necesidad y la lógica del mercado en la producción de la ciudad popular. Proyecto Guayaquil Ecológico. En 02 Economía urbana y gobernanza.
Memorias del Congreso de Estudios de la ciudad. Cuenca: Universidad del Azuay. Recuperado de http://revistas.uazuay.edu.ec/index.php/ memorias/issue/view/19/PDF-CITIVIC-02

Schiavo, E., Vera, P. y Nogueira, C. (2014). Los movimientos sociales y formas de urbanización metropolitana en el marco del posneoliberalismo. El caso de GIROS en la ciudad de Rosario, Argentina. Revista Quid 16, (3), 158-69.

SENPLADES. (2009). Plan nacional para el buen vivir 2009-2013 versión resumida Quito: Autor.

Slater, T. (2006). The eviction of critical perspectives from gentrification research. International Journal of Urban and Regional Research, 30(4), 737-57. https://doi.org/10.1111/j.1468-2427.2006.00689.x

Smith, N. (1996). The new urban frontier: Gentrification and the revanchist city. London: Routledge.

Stoessel, S. (2014). Giro a la izquierda en la América Latina del siglo XXI. Revisitando los debates académicos. Polis, 13(39), 2-18. http://dx.doi.org/10.4067/S0718-65682014000300007

Taylor, S. y Bogdan, R. (1987). Introducción a los métodos cualitativos de investigación. Barcelona: Paidós.

Zibechi, R. (2008). Territorios en resistencia: cartografía politica de las periferias urbanas latinoamericanas. Buenos Aires: Lavaca. 


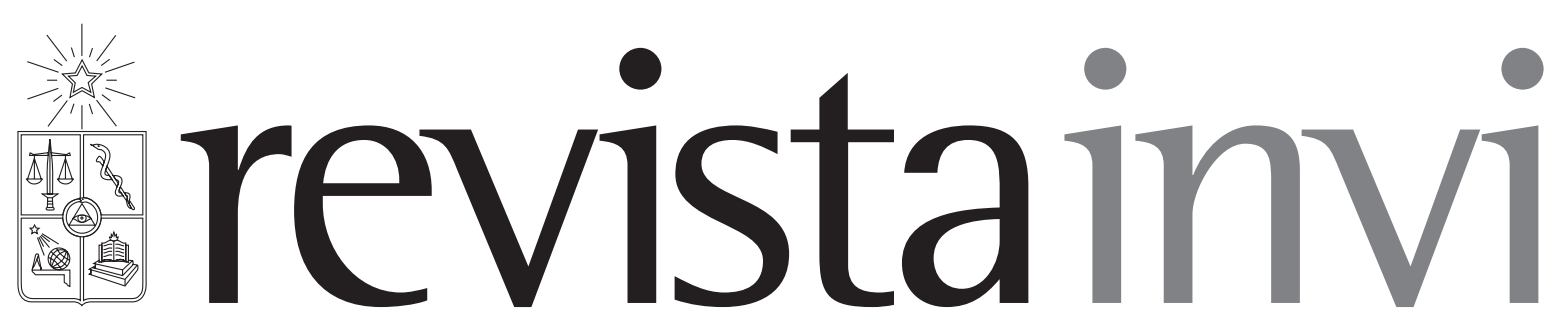

Revista INVI es una publicación periódica, editada por el Instituto de la Vivienda de la Facultad de Arquitectura y Urbanismo de la Universidad de Chile, creada en 1986 con el nombre de Boletín INVI. Es una revista académica con cobertura internacional que difunde los avances en el conocimiento sobre la vivienda, el hábitat residencial, los modos de vida y los estudios territoriales. Revista INVI publica contribuciones originales en español, inglés y portugués, privilegiando aquellas que proponen enfoques inter y multidisciplinares y que son resultado de investigaciones con financiamiento y patrocinio institucional. Se busca, con ello, contribuir al desarrollo del conocimiento científico sobre la vivienda, el hábitat y el territorio y aportar al debate público con publicaciones del más alto nivel académico.

Director: Dr. Ricardo Tapia Zarricueta, Universidad de Chile, Chile.

Editor: Dr. Luis Campos Medina, Universidad de Chile, Chile.

Editor asistente: Dr. Walter Imilan, Universidad de Chile, Chile.

Coeditora: Srta. Sandra Rivera, Universidad de Chile, Chile.

\section{COMITÉ EDITORIAL:}

Dr. Victor Delgadillo, Universidad Autónoma de la Ciudad de México, México.

Dra. María Mercedes Di Virgilio, CONICET/ IIGG, Universidad de Buenos Aires, Argentina.

Dra. Irene Molina, Uppsala Universitet, Suecia.

Dr. Gonzalo Lautaro Ojeda Ledesma, Universidad de Valparaíso, Chile.

Dra. Suzana Pasternak, Universidade de São Paulo, Brasil.

Dr. Javier Ruiz Sánchez, Universidad Politécnica de Madrid, España.

Dra. Elke Schlack Fuhrmann, Pontificia Universidad Católica de Chile, Chile.

Dr. Carlos Alberto Torres Tovar, Universidad Nacional de Colombia, Colombia.

Sitio web: http://www.revistainvi.uchile.cl/

Correo electrónico: revistainvi@uchilefau.cl

Licencia de este artículo: Creative Commons Atribución-Compartirlgual 4.0 Internacional (CC BY-SA 4.0) 